\title{
Brüssel: Podiumsdiskussion zu Datenschutz und Wettbewerb am 10. Juli 2019
}

Vor mehr als 300 Gästen diskutierte am 10. Juli 2019 ein hochkarätig besetztes Panel über die Herausforderungen für Datenschutz und Wettbewerbsfähigkeit im digitalen Zeitalter. Auf Initiative des Europäischen Datenschutzbeauftragten (EDPS) und des Bundesbeauftragten für den Datenschutz und die Informationsfreiheit (BfDI) wurden unter anderem die Möglichkeiten der Zusammenarbeit zwischen den Regulierungsbehörden und die Herausforderungen neuer Technologien für einen effektiven Datenschutz diskutiert.

Der Europäische Datenschutzbeauftragte Giovanni Buttarelli erklärte: »Die massenhafte Datenverarbeitung hat schwerwiegende Folgen nicht nur für den Einzelnen, sondern auch für die Gesellschaft, die Demokratie und die Umwelt. Daten sind zu einem geostrategischen Faktor geworden, weil die Unterschiede in der digitalen Dividende, also der Aufteilung der Gesellschaft zwischen denjenigen, die die Macht über ihr digitales Leben, ihre Freiheiten und ihre Privatsphäre haben, und denjenigen, die keine Macht haben, immer weiter zunehmen.«

Die Auswirkungen von Datenkonzentration und Datenmacht auf Bürgerrechte und Wettbewerbsfreiheit gehen mittlerweile weit über die Reichweite einzelner Rechtsgebiete, wie den Datenschutz, hinaus. Nicht zuletzt deshalb ist der Datenschutz nicht mehr nur ein Thema für die Datenschutz-aufsicht, sondern längst auch für weitere Regulierungsbehörden, zum Beispiel im Verbraucherschutz- oder Wettbewerbsrecht. Um der faktischen Datenmacht der großen global operierenden Technologieunternehmen zu begegnen, bedarf es daher einer stärkeren Zusammenarbeit zwischen den verschiedenen Aufsichtsbehörden. Die Notwendigkeit eines starken gesamteuropäischen Auftretens bei Daten-schutzfragen zeigt sich auch bei der Betrachtung neuer Technologien wie Künstlicher Intelligenz (KI), Cloud Services oder Blockchain.
Der Bundesbeauftragte für den Datenschutz und die Informationsfreiheit, Ulrich Kelber, stellte hierzu fest: »Neue Technologien schaffen Chancen - gleichzeitig aber auch Risiken, insbesondere für die informationelle Selbstbestimmung. Daher müssen wir gemeinsam daran arbeiten, proaktiv im Sinne der Gewährleistungsziele des Datenschutzes von Anfang an bei einer »menschzentrierten « Technikgestaltung mitzuwirken. Dabei sollten wir das Modell der rechtsgebietsübergreifenden Behördenkooperation nicht nur auf nationaler Ebene, sondern auch auf EU-Ebene stärker institutionalisieren."

Sowohl Buttarelli als auch Kelber waren sich einig, wie wichtig es ist, dass durch die DSGVO garantierte hohe Datenschutzniveau als globalen Maßstab für die Entwicklung neuer Technologien zu etablieren. Der Datenschutz im All-gemeinen und die DSGVO im Besonderen sollten nicht als Wettbewerbsnachteil für europäische Unternehmen angesehen werden. Datenschutz ist wesentlicher Bestandteil einer nachhaltigen KI-Technologie. Gerade im Bereich der digitalen Innovation kann ein hohes Datenschutzniveau sogar ein Wettbewerbsvorteil sein.

Neben dem EDPS und dem BfDI nahmen auch der Präsident des Bundeskartellamtes, Andreas Mundt, die Präsidentin der französischen Datenschutzbehörde (CNIL), Marie-Laure Denis, der Generalsekretär der Europäischen Kommission, Martin Selmayr, und die britische Informationskommissarin, Elizabeth Denham, an der Podiumsdiskussion teil.

Giovanni Buttarelli, der Europäischen Datenschutzbeauftragte (EDPS) und

Ulrich Kelber, der Bundesbeauftragte für den Datenschutz und die Informationsfreiheit (BfDI) 